\title{
Intraductal Cribriform Breast Adenocarcinoma
}

National Cancer Institute

\section{Source}

National Cancer Institute. Intraductal Cribriform Breast Adenocarcinoma. NCI Thesaurus. Code C5138.

A ductal carcinoma in situ of the breast characterized by the presence of a cribriform architectural pattern. 